\title{
Rank ordering multifractal analysis of the auroral electrojet index
}

\author{
G. Consolini ${ }^{1}$ and P. De Michelis ${ }^{2,3}$ \\ ${ }^{1}$ INAF-Istituto di Fisica dello Spazio Interplanetario, 00133 Roma, Italy \\ ${ }^{2}$ Istituto Nazionale di Geofisica e Vulcanologia, 00143 Roma, Italy \\ ${ }^{3}$ Dip. Scienze della Terra, Università degli Studi di Siena, 53100 Siena, Italy
}

Received: 28 January 2011 - Revised: 13 April 2011 - Accepted: 22 April 2011 - Published: 6 May 2011

\begin{abstract}
In the second half of the 90 s interest grew on the complex features of the magnetospheric dynamics in response to solar wind changes. An important series of papers were published on the occurrence of chaos, turbulence and complexity. Among them, particularly interesting was the study of the bursty and fractal/multifractal character of the high latitude energy release during geomagnetic storms, which was evidenced by analyzing the features of the Auroral Electrojet (AE) indices. Recently, the multifractal features of the small time-scale increments of AE-indices have been criticized in favor of a more simple fractal behavior. This is particularly true for the scaling features of the probability density functions (PDFs) of the AE index increments. Here, after a brief review of the nature of the fractal/multifractal features of the magnetospheric response to solar wind changes, we investigate the multifractal nature of the scaling features of the AE index increments PDFs using the Rank Ordering Multifractal Analysis (ROMA) technique. The ROMA results clearly demonstrate the existence of a hierarchy of scaling indices, depending on the increment amplitude, for the data collapsing of PDFs relative to increments at different time scales. Our results confirm the previous results by Consolini et al. (1996) and the more recent results by Rypdal and Rypdal (2010).
\end{abstract}

\section{Introduction}

Over the last two decades there has been a steady increase in the investigation of the nonlinear response of the Earth's magnetosphere to solar wind changes. Mainly, these studies have been focused on the magnetospheric response during magnetic storms and substorms, and have been essentially carried out analyzing time series of some geomagnetic indices, for example the auroral electrojet $\mathrm{AE}$ indices or the Dst index. These indices can be indeed considered as proxies of magnetospheric processes related to the occurrence of storms and substorms, and allow us to monitor some of the most relevant current systems, which are activated during magnetic storms and substorms.

Among them the AE-indices (AU, AL, AO, and AE), introduced by Davis and Sugiura (1966), are certainly a convenient proxy of the magnetospheric substorm activity. In particular, the AE index describes the total intensity of two electrojets (eastward and westward), which are almost permanently observed in the auroral ionosphere and are enhanced during magnetic auroral substorms. For this reason, this index has been usefully employed both qualitatively and quantitatively as a correlative index in studies of substorm morphology and of coupling between the interplanetary magnetic field and the Earth's magnetosphere.

In this framework, a first study was done by Tsurutani et al. (1990) at the beginning of 90s. The authors compared the power spectra of the AE index and of the southward component $B_{z}$ of the Interplanetary Magnetic Field (IMF) for the same time interval, demonstrating that the Earth's magnetosphere response to the solar wind energy input was neither periodic nor quasiperiodic, and that the magnetosphere acted essentially as a low pass filter on the IMF spectrum. This result suggested that the magnetospheric response to the solar wind might be nonlinear.

Successively, the analyses of the AE index seemed to indicate that the magnetospheric disturbance data had a low correlation dimension in the range between 2.4 and 4.2 (Vassiliadis et al., 1990; Sharma et al., 1993; Takalo et al., 1993) suggesting that the dissipative organized response of the magnetosphere to the solar wind input might be described by a low-order system of equations. It was proposed that the global geomagnetic system was a low dimensional, possibly chaotic, nonlinear system.

\section{Correspondence to: G. Consolini}

(giuseppe.consolini@ifsi-roma.inaf.it)

Published by Copernicus Publications on behalf of the European Geosciences Union and the American Geophysical Union. 
In the same period, it was also investigated if the dynamics of the magnetosphere could be described in terms of stochastic colored or bicolored noise (Shan et al., 1991; Roberts, 1991; Pavlos et al., 1992). Takalo and coauthors, in an important series of works (Takalo et al., 1993, 1994; Takalo and Timonen, 1994), studied this hypothesis extensively, concluding that a stochastic process, not a chaotic one, generated $\mathrm{AE}$ data. A primary motivation in the careful search for low-dimensional features in the magnetospheric activity was the hope of becoming capable of constructing physically relevant low-dimensional models for magnetospheric dynamics. These models could be of great value for understanding the solar wind-magnetosphere-ionosphere interaction and for forecasting the magnetosphere response to changing solar wind conditions. However, soon after the first estimates of the correlation dimension of the magnetosphere, Prichard and Price $(1992,1993)$ showed that the estimates of low dimension of the magnetosphere were caused by the long autocorrelation times of the system rather than by the low dimensional magnetospheric dynamics. Consequently, there was no evidence for the presence of a low-dimensional attractor in the AE data that they had studied.

Later on, Consolini et al. (1996) investigated the possible multifractal nature of the AE index and recognized the necessity of introducing a hierarchy of dimensions (the Renyi dimensions $D_{q}$ ) to characterize the intermittent character of the small-scale increments (sometime named as fluctuations) of this index. This result suggested that turbulence had to be considered as a much more relevant phenomenon than lowdimensional chaos in the magnetospheric dynamics. Consequently, the magnetospheric dynamics seemed to be characterized by many degrees of freedom and/or by stochastic fluctuations. Furthermore, the multifractal character of the AE index increments indicated that intermittency might play a relevant role in dissipation mechanisms related to the auroral electrojet. The occurrence of intermittency was successively confirmed by the departures of the probability distribution functions (PDFs) of the AE index small time-scale increments from the Gaussian shape both in quiet and disturbed periods (Consolini and De Michelis, 1998). We note that nowadays the crucial role of turbulence in several magnetospheric/ionospheric phenomena/processes is well documented (e.g., Zimbardo et al., 2010).

Approximately in the same period, the near-criticality dynamics of magnetospheric response during magnetic substorms, as early hypothesized by Chang (1992), was evidenced by investigating the power-law distribution for the AE index activity bursts (Consolini, 1997, 2002) and its low frequency stochastic variations of the $1 / f^{\beta}$ power spectrum (Uritsky and Pudovkin, 1998). Successively, several attempts were made to model such a near-criticality behavior by means of Self-Organized Criticality models (e.g., Chapman et al., 1998; Consolini and De Michelis, 2001). Based on the assumption that the observed scaling invariant distribution of the AE index burst sizes might be the counterpart of sporadic reorganization processes among multiscale magnetic coherent structures near a critical state, Chang (1999) coined the term Forced and/or Self-Organized-Criticality.

Coming back to the multifractal features of the AE index, in the last decade some works (Hnat et al., 2002, 2005; Chapman et al., 2005; Watkins et al., 2005) have suggested, in contrast with previous papers, that the auroral electrojet time series present a very weak multifractality and that the multifractal features of the AE index variations may be an artifact of statistically poorly resolved behavior of the largest fluctuations. It has also been suggested that the AE index can be modeled as a fractional $\alpha$-stable motion (also called fractional Lévy flight) on time scales $<10^{2}$ min (Watkins et al., 2005).

Recently, the possible role that stochastic fluctuations may play in the AE index fluctuations during magnetospheric substorms, has been carefully investigated (Pulkkinen et al., 2006; Anh et al., 2008). The results of these studies have again suggested that the description of the complex nature of $\mathrm{AE}$ index requires to adopt novel approaches based on fractional calculus.

In this framework, where the multifractality of the AE index variations remains an open issue, a work by Rypdal and Rypdal (2010) has been recently introduced. In their paper, the authors explore the implications of modeling the AE index as a smoothly truncated Lévy flight and demonstrate that although such processes seem to capture some of the properties of the AE index, there are some inconsistencies which lead the authors to believe that the multifractal model provides a more accurate description of the $\mathrm{AE}$ index variations.

In this paper we investigate the scaling collapse of the PDFs of AE index increments at different time scales using the approach based on the Rank-Ordered Multifractal Analysis (ROMA) introduced by Chang and Wu (2008) (see also Chang et al., 2010, for a review). This method will allow us to analyze and confirm the multifractal characteristics of the auroral electrojet AE index, thus supporting all previous findings related to the occurrence of time-intermittency, turbulence and/or criticality in the magnetospheric dynamics in response to solar wind changes.

This paper is structured as follows: in Sect. 2, we briefly review the concept of multifractality and introduce the ROMA technique; in Sect. 3 we proceed to explore how test the existence of a multifractal structure of the AE index increments applying such technique to more than $10 \mathrm{yr}$ data of the AE index; finally in Sect. 4 we discuss and summarize our results.

\section{Multifractality and ROMA: a brief introduction}

The concept of multifractal set and the term multifractal measure date back more than $30 \mathrm{yr}$, and have been extensively applied to model the anomalous scaling features, which are responsible for intermittency in the framework of fully 
developed turbulence (see e.g. Mandelbrot, 1974; Benzi et al., 1984; Frisch and Parisi, 1985; Halsey et al., 1986; Paladin and Vulpiani, 1987) and space plasma turbulence (see e.g. Burlaga, 1991a,b; Carbone, 1993; Marsch et al., 1996; Macek et al., 2005). According to the traditional representation "a multifractal measure can be thought as the union of a continuous infinity of intertwined sets, each of which is an infinitesimal unifractal measure, characterized by a single value of the Hölder scaling exponent $\alpha$ and supported by a fractal set of dimension $f(\alpha)$ "'(Mandelbrot, 1989, p. 23).

A slightly different definition of multifractality was provided by Mandelbrot (1989): "In one phrase, the generalization from fractal sets to multifractal measures involves the passage from geometric objects characterized primarily by one number, to geometric objects characterized primarily by a function. This function can be a probability distribution that has been renormalized and plotted suitably. In a different single phrase, the generalization from fractal sets to multifractal measures involves the passage from a finite number of fractal dimensions to an infinite number of dimensions".

One of the traditional approaches to multifractal measure $\epsilon(x)$ (see e.g. Paladin and Vulpiani, 1987) consists of introducing an appropriate partition $\{\delta x\}$ of the measure, of defining a coarse-grained weight $p_{i}(\delta x)$, and of investigating the scaling features of the associated partition function $Z_{q}(\delta x)$ of moment order $q$ (Paladin and Vulpiani, 1987). The presence of an anomalous scaling of the scaling exponents $\gamma(q)$ of the $Z_{q}(\delta x)$ as a function of $q$ i.e., $\gamma(q) \sim D_{q}(q-1)$ with $D_{q}$ a convex function of $(q)$ is the signature of the multifractal nature of the measure $\epsilon(x)$. As a consequence of the anomalous behavior of the scaling exponents $\gamma(q)$, the description of the measure distribution over the support requires a hierarchy of fractal dimensions.

In the framework of fully developed turbulence the characterization of the multifractal nature of the velocity field is generally studied by analyzing the scaling features of the structure function of order $q, S_{q}\left(\delta r_{\|}\right)$, of the longitudinal velocity increments at the scale $\delta r_{\|}$(Frisch, 1995). In general, given a signal $x(t)$, we can define a generalized structure function of order $q$ as

$S_{q}(\tau)=\left\langle|x(t+\tau)-x(t)|^{q}\right\rangle$.

For self-similar signals the generalized structure function $S_{q}(\tau)$ is expected to scale according to a power law (Mandelbrot, 1989; Paladin and Vulpiani, 1987; Frisch, 1995),

$S_{q}(\tau) \sim \tau^{\zeta(q)}$,

with scaling exponent $\zeta(q)$. For mono-fractal signals the scaling exponent $\zeta(q)$ is generally a linear function of the moment order $q$, so that the knowledge of a single number $H=\zeta(q) / q$ (named Hurst exponent) is sufficient to characterize the scaling features of the signal $x(t)$. However, there are situations where the dependence of $\zeta(q)$ on the moment order $q$ is not linear. Sometimes, the dependence of $\zeta(q)$ on the moment order $q$ is indeed a convex function, i.e., it displays an anomalous scaling. In this case, one single number is no longer sufficient to characterize the scaling features of the signal $x(t)$, but a hierarchy of scaling exponents $H$ is necessary. This behavior mirrors the complex nature of the scaling features of the signal $x(t)$, which is related to its multifractal nature. In the framework of fully developed turbulence the emergence of an anomalous scaling of the scaling exponents $\zeta(q)$ on the moment order $q$ is read as a signature of intermittency, which is also related to the departure from a Gaussian statistics of the longitudinal velocity increments at the smallest scale.

All the previous properties are also reflected in the statistics of the signal increments, $\delta x(\tau)=x(t+\tau)-x(t)$, at different scales. Given a signal $x(t)$, it is indeed possible to evaluate the probability distribution function $P(\delta x ; \tau)$ of signal increments $\delta x(\tau)$ at the time scale $\tau$, which is generally scale dependent. For some classes of signals it is possible to construct a master curve by simply performing a scale transformation,

$$
\left\{\begin{array}{l}
\delta x \\
P(\delta x ; \tau) \longrightarrow \tau^{-s} \delta x \\
\tau^{s} P(\delta x ; \tau)=P_{s}\left(\tau^{-s} \delta x\right)
\end{array}\right.
$$

where $s$ is an appropriate scaling exponent. In this case, all the PDFs at different time scales will collapse into an invariant scaling function, the master curve $P_{s}\left(\tau^{-s} \delta x\right)$, (Chang et al., 1973) and the observed behavior will be monofractal. Nevertheless, it is not always possible to get a single master curve by using one-exponent scaling. Generally, a continuous change of the shape of the PDFs from small scales (where PDFs are generally leptokurtotic) to large ones (where the statistics is more or less Gaussian) occurs. The absence of a master curve is the signature of the multifractal nature of the signal increments. What happens is that the scaling exponents $s$ may depend on the amplitude of the increments, so that increments of different sizes may be characterized by different scaling exponents. This idea is contained in ROMA to study the multifractal nature of a signal (Chang and Wu, 2008; Tam et al., 2010; Chang et al., 2010).

The core of the rank-ordered multifractal analysis resides in exploring the singular nature of the fluctuations (increments) by grouping them according to the scaled-size (Chang and $\mathrm{Wu}, 2008$ ). This way to proceed is equivalent to reshuffling the problem related to the dominant population of small-amplitude increments. Indeed, the traditional methods of investigating multifractality (based on partition function approach, structure function scaling features, statistical moments of PDFs, etc.) are based on the statistics of the full set of the signal increments, which naturally implies that small size increments, being generally the most probable, largely dominate the large size ones, which are by far the less numerous. This may affect the results and, furthermore, it can be difficult to provide a good physical interpretation from the simple examination of the anomalous scaling of the exponents. 
The idea of ROMA is to partition the domain of the rescaled increments $\left(Y=\tau^{-s} \delta x\right)$ into separate ranges, and for each range to determine the value of the scaling exponent $s$ that satisfies the following equation

$\tau^{s} P(\delta x ; \tau)=P_{s}\left(\tau^{-s} \delta x\right)$.

Consequently, it will be possible to find a hierarchy of scaling indices $s$ associated with the different ranges of $Y$. To find the scaling exponent $s$ for a given range of $Y\left(Y \in\left[Y_{i}, Y_{i+1}\right]\right)$ one has to solve the following functional equation for a range-limited structure function $S_{q}^{\prime}(\tau)$,

$S_{q}^{\prime}(|\delta x| ; \tau)=\int_{\tau^{s} Y_{i}}^{\tau^{s} Y_{i+1}}|\delta x(\tau)|^{q} P(|\delta x|, \tau) d|\delta x| \simeq \tau^{q s}$

without making any distinction between positive and negative increments (thus assuming a symmetry in the PDF). If a single solution exists for $s$ in the chosen $Y$-range, then the increments will be characterized by a mono-fractal behavior with a local Hurst exponent $s$ in this range.

\section{Data description and results}

To analyze the multifractal and intermittency features of the auroral electrojet (AE) index we consider a continuous time series with a time resolution of $1 \mathrm{~min}$ covering a period of about $10.5 \mathrm{yr}$ from 1 January 1978 to 30 June 1988 for a total amount of points of the order of $6 \times 10^{6}$ points. Data come from both the National Geophysical Data Center (NGDC, Boulder, Colorado) - available on a CD-Rom - and the World Data Center I (Kyoto, Japan). The choice of such a long time series without any additional assumption is motivated by two different arguments

- to have a good statistics for our analysis,

- to consider a period of the order of the solar cycle to cover all the possible configurations of the state space of the magnetospheric system.

As discussed in the Introduction it is known that $\mathrm{AE}$ index is characterized by a power-law spectrum, $S(f) \sim f^{-\beta}$, with $\beta \sim-2$ for periods in the interval $[2,240]$ min (see e.g. Tsurutani et al., 1990; Consolini, 2002). For this reason we limit our analysis of the scaling features to time scales shorter than $100 \mathrm{~min}$.

In the analysis of the scaling features of the PDFs of the $\mathrm{AE}$ index increments $(\delta \mathrm{AE}(\tau)=\mathrm{AE}(t+\tau)-\mathrm{AE}(t))$, our first step is to investigate the existence of a single scale exponent, as already done by other authors on different time intervals (Hnat et al., 2002, 2005). We remark that the computation of increments $\delta \operatorname{AE}(\tau)$ at the temporal scale $\tau$ is made using disjoint time intervals (i.e., not using a moving window technique). Furthermore, to compute the PDFs we utilize

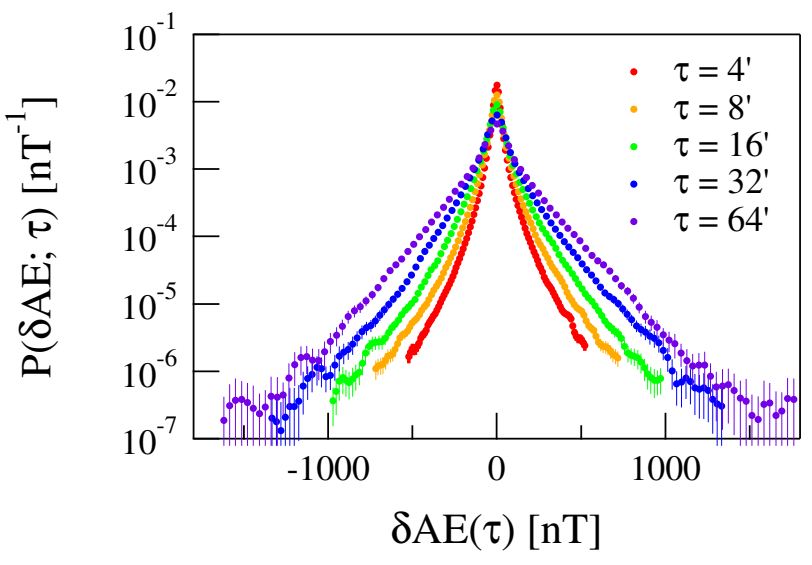

Fig. 1. The PDFs $P(\delta \mathrm{AE} ; \tau)$ of the $\mathrm{AE}$ index increments $\delta \mathrm{AE}(\tau)$ for $\tau=4,8, \ldots, 64 \mathrm{~min}$.

a non-parametric estimation method based on kernel techniques (Kaiser and Schreiber, 2002),

$P(\delta \mathrm{AE} ; \tau)=\frac{1}{N \epsilon} \sum_{n=1}^{N} K\left(\frac{\delta \mathrm{AE}-\delta \mathrm{AE}(n)}{\epsilon}\right)$

where $\delta \mathrm{AE}(n)$ is the actual AE-increment time series, $\epsilon$ is the bin width (chosen as $0.2 \sigma$ where $\sigma$ is the standard deviation of $\delta \mathrm{AE})$ and $K(u)$, where $u=(\delta \mathrm{AE}-\delta \mathrm{AE}(n)) / \epsilon$, is the kernel that we choose to be of a Gaussian type

$K(u)=\frac{1}{\sqrt{2 \pi}} \exp \left(-\frac{1}{2} u^{2}\right)$.

The reason why we estimate the PDFs by means of the above technique is the attempt to reduce as much as possible the effects of errors coming from the discrete nature of the AE index. Error bar estimation is done assuming a Poissonian distribution of points in $|u| \leq 1$, i.e., $\delta P(\delta \mathrm{AE}, \tau) \sim$ $\sqrt{N_{u}} / N \epsilon$, with $N_{u}$ the number of points in the range $|u| \leq 1$. This is a conservative assumption for the error bar estimation.

Figure 1 reports the PDFs $P(\delta \mathrm{AE} ; \tau)$ of the AE index increments $\delta \operatorname{AE}(\tau)$ at some different time scales $\tau(\tau=$ $4,8, \ldots, 64 \mathrm{~min})$.

According to the procedure introduced by Hnat et al. (2002), we look for a single-scaling exponent $s$ studying the probability of return $P(0 ; \tau)$ as a function of $\tau$. This quantity is indeed expected to scale as

$P(0 ; \tau) \sim \tau^{-s}$,

where $s$ is the scaling exponent. Figure 2 shows the trend of the probability of return $P(0 ; \tau)$ plotted versus $\tau$ in the time domain $[4 ; 64]$ min on a log-log diagram. A very good power law dependence of $P(0 ; \tau)$ on $\tau$ is found and the obtained value of the scaling exponent $s=[0.487 \pm 0.002]$ is quite well in agreement with the previous results (Hnat et al., 2002). We note that although the observed scaling exponent is very close to that of a Gaussian process $(s=1 / 2)$, in 


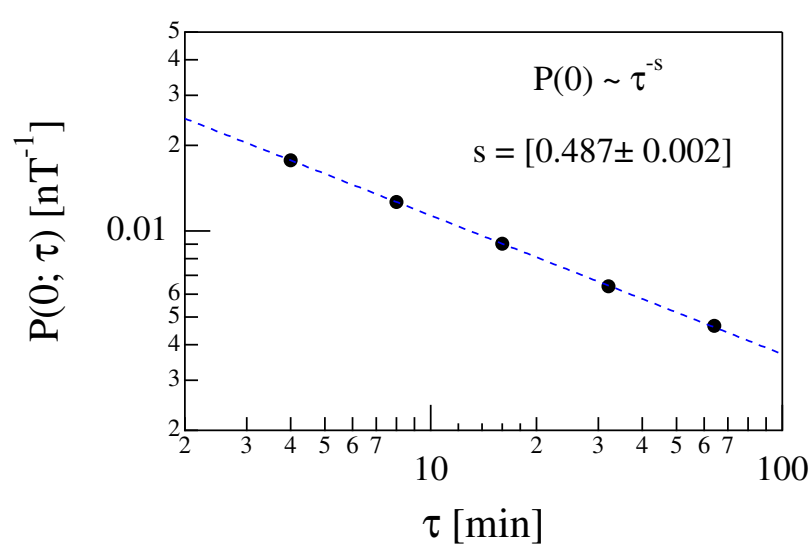

Fig. 2. The probability of return $P(0 ; \tau)$ versus $\tau$ on a log-log plot. The dashed line is a power-law with exponent $s \sim 0.487$.

our case we are clearly in presence of a non-Gaussian process as well documented by the shape of the PDFs. A possible origin of such a value could be related to the relevance that stochastic Gaussian noise could play in the case of small amplitude $\ll \sigma_{\delta \mathrm{AE}}$ increments. In other words, the observed PDFs could be the superposition of two distribution a more or less Gaussian distribution for very small values of the increments and a leptokurtotic one for large increments.

We attempt to collapse all the PDFs onto a single universal master curve moving from the scale-transformation of Eq. (3) within the selected range of $\tau \mathrm{s}$. Figure 3 shows the results, where $Y$ is the scaled variable, defined as

$Y=\left(\frac{\tau}{\tau_{0}}\right)^{-s} \delta \mathrm{AE}(\tau)$

where $\tau_{0}$ is a reference time scale (here we choose $\tau_{0}=60 \mathrm{~s}$ ). We remark that according to the previous definition, the scaled increments $Y$ are measured in nT. Data collapsing is very poor for $|Y|>100 \mathrm{nT}$. It is not possible to use a single exponent to collapse all the PDFs onto a single master curve, and consequently the nature of the AE index is not simply monofractal, as stated by Hnat et al. (2002) using a different dataset. A possible explanation of the observed discrepancy between our results and those of Hnat et al. (2002) could be the different dataset used in the analysis.

Taking into account this result, we attempt to collapse each corresponding unscaled PDF onto a single master curve using the ROMA technique. Because there is no reason to assume that the PDFs of the AE index increments are symmetric with respect to $\delta \mathrm{AE}=0$, we investigate the scaling features of both positive and negative $\delta \mathrm{AE}$, modifying Eq. (5) as follows,

$S_{q}^{\prime}(\delta x ; \tau)=\int_{\tau^{s} Y_{i}}^{\tau^{s} Y_{i+1}}|\delta x(\tau)|^{q} P(\delta x, \tau) d \delta x$,

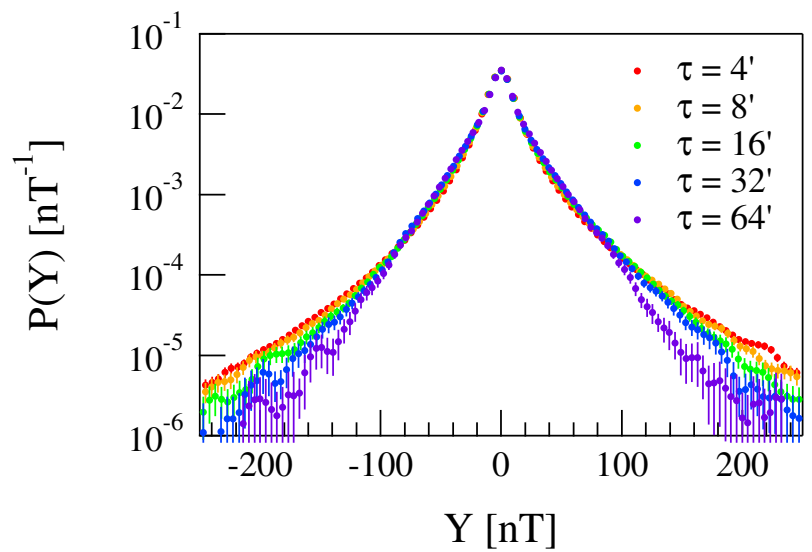

Fig. 3. Collapsing of the PDFs of the AE index increments $\delta \mathrm{AE}(\tau)$ for $\tau=4,8, \ldots, 64 \mathrm{~min}$, scaled according to Eq. (9). The scaling exponent is $s=0.487$. Here $P(Y)$ and $Y$ stand for the scaled PDF and $\delta \mathrm{AE}$, respectively.

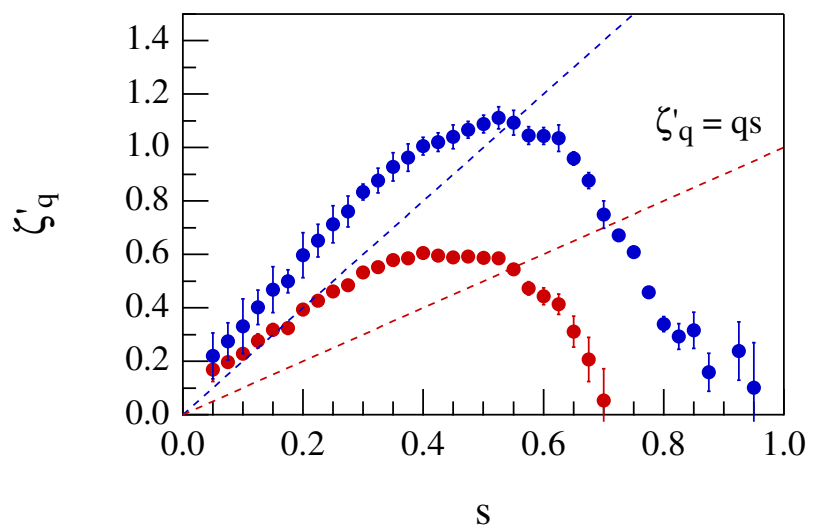

Fig. 4. Plot of $\zeta^{\prime}(q)$ vs. $s$ for the determination of the scaling parameter $s$ in the range $Y \in[45,55] \mathrm{nT}$ for $q=1$ (red circles) and $q=2$ (blue circles), respectively. The two dashed lines refer to $\zeta^{\prime}(q)=q s$ with $q=1$ (red) and $q=2$ (blue), respectively.

where $Y_{i}$ is a positive/negative real number $\left(Y_{i} \in \mathcal{R}\right)$, and looking for a solution of the type,

$S_{q}^{\prime}(\delta x ; \tau) \simeq \tau^{\zeta^{\prime}(q)}, \rightarrow \zeta^{\prime}(q)=q s$

where $s$ is the local Hurst exponent $(s \equiv H \in[0,1])$. The analysis of rank-ordered structure function is limited to the range $Y \in[-205,205] \mathrm{nT}$, using a regular partition of width $\Delta Y=10 \mathrm{nT}$. The reason for limiting our analysis to this $Y$ range is the necessity of correctly estimating the probability density function at large values of the scaled variable $Y$ especially for large time intervals $\tau$.

Figure 4 reports an example of the application of ROMA to the range of $Y \in[45,55] \mathrm{nT}$ for the moment orders $q=1$ and $q=2$, respectively. In this selected $Y$ range, we find a single solution $s(q)$, which satisfies Eq. (11), for each $q$ value. This solution is given by the intersection of the expected dependence of $\zeta_{q}^{\prime}=q s$ and the actual trend of $\zeta_{q}^{\prime}(s)$. 


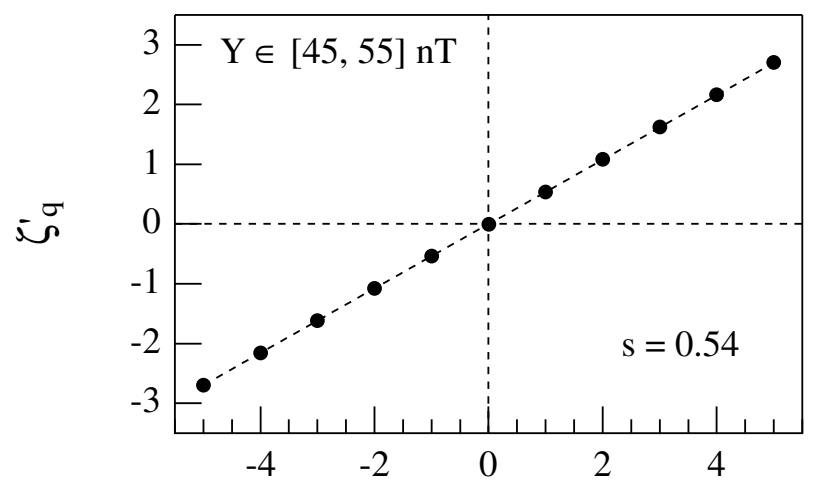

q

Fig. 5. Plot of $\zeta^{\prime}(q)$ vs. $q$ to confirm the mono-fractal scaling with scaling exponent $s=0.54$ in the range $Y \in[45,55] \mathrm{nT}$. The dashed line is the line $\zeta^{\prime}(q)=0.54 q$.

The presence of a single solution $\left(s=s_{1}=s_{2}=0.54\right.$ where $s_{q}$ is the solution for the moment order $q$ ) independent from $q$ suggests that a monofractal scaling characterizes the selected $Y$ range. This hypothesis is confirmed in Fig. 5 where we report the solutions of $\zeta^{\prime}(q)$ obtained by solving numerically Eq. (11) for $q \in[-5,5]$, and compare them with $\zeta^{\prime}(q)=q s$. The excellent linear scaling of $\zeta^{\prime}(q)$ is the signature for a mono-fractal behavior of $\delta \mathrm{AE}$ in the selected $Y$ range.

Similarly, we look for solutions of Eq. (11) for other $Y$ ranges, thus constructing the rank-ordered spectrum, $s=$ $s(Y)$, of the scaling indices. We find single solutions for each range of $Y$ except for the case of $Y \in[-45,+45]$ nT where a secondary solution is obtained. This extra solution, that can be considered as a secondary branch for $Y \in[-45,45] \mathrm{nT}$, does not satisfy the requirement of linear scaling with the moment order $q$, as clearly shown in Fig. 6 for a particular $Y$ interval. For this reason we consider these extra solutions as spurious ones and neglect them in what follows.

Figure 7 shows the rank-ordered spectrum $s(Y)$ for both positive and negative values of $Y$. The rank-ordered spectrum $s(Y)$ can be considered as a discrete version of a continuous multifractal spectrum for the $\mathrm{AE}$ index increments. The presence of a certain degree of asymmetry is observed among the scaling exponents of positive and negative AE index increments in the range $Y \in[-25,25] \mathrm{nT}$. This asymmetry has to be related to the physical mechanism of auroral electrojet current increase and decay. We note that the scaling exponent $s$ approaches neither 0.5 for $Y \rightarrow 0$, as it would be expected in the case of a Gaussian random process, nor the scaling exponent of the probability of return $P(0 ; \tau)$. It tends to $s(0) \sim 0.685$. The finite size of the $Y$ range $(Y \in[-5,5]$ nT, i.e., $\left.\Delta Y=Y_{\max }-Y_{\min }=10 \mathrm{nT}\right)$ used to determine $s(0)$ could explain this result. To check this point we reduce the size of the $Y$ range considered to determine $s(0)$, finding that $s(0) \rightarrow 0.49$ for $\Delta Y=4$ (see Table 1). This value is well in

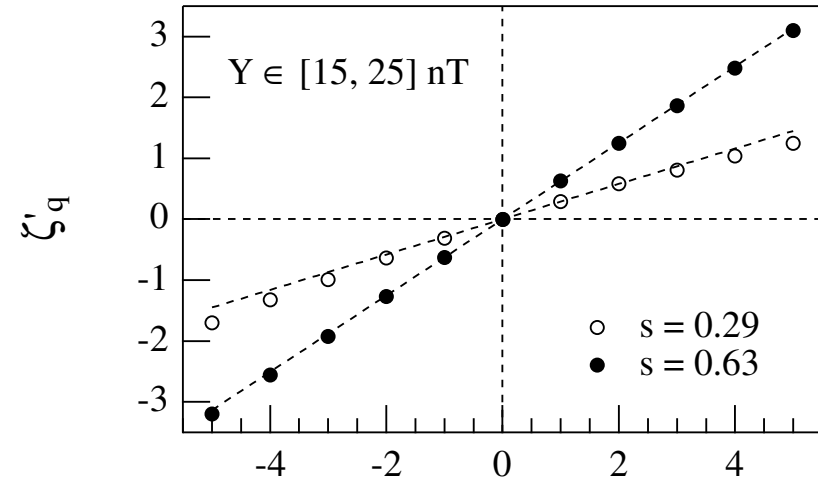

q

Fig. 6. Plot of $\zeta^{\prime}(q)$ vs. $q$ for the two solutions found in the interval $Y \in[15,25] \mathrm{nT}$. While one solution (solid circle) shows the linear dependence on $q$, as expected for a mono-fractal scaling, the second one (empty circle) displays a clear anomalous dependence on $q$.

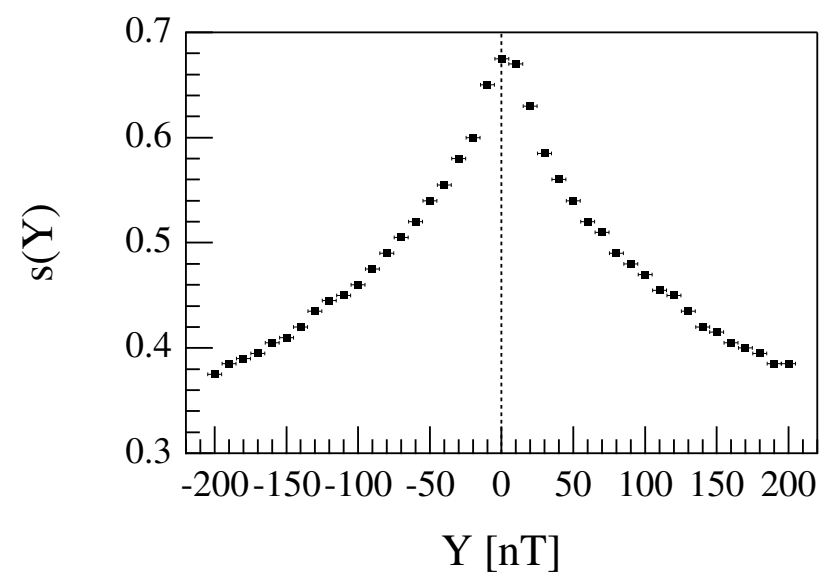

Fig. 7. Profile of the rank-ordered spectrum $s(Y)$ for scaling exponents.

agreement with the single scaling exponent found by us in our previous analysis and consistent with Hnat et al. (2002).

Another peculiar feature of the rank-ordered spectrum $s(Y)$ is the decrease of $s$ for increasing $Y$, suggesting a less space filling character of large scaled increments $Y$. In other words, this result reflects the sparse nature in time of the occurrence of large amplitude increments.

Taking into account that the scaling exponent $s(Y)$ is equivalent to the Hurst exponent $H$ for the $Y$ scaled increments and that the Hurst exponent $H$ is related to the degree of persistency (Hergarten, 2002), the rank-ordered spectrum $s(Y)$ could consequently reflect the different persistency degree of small and large (negative and positive) scaled increments $Y$. In particular the rank-ordered spectrum $s(Y)$, reported in Fig. 7, seems to suggest that the small AE index scaled increments $(|Y|<70 \mathrm{nT})$ are persistent $(s>1 / 2)$, while very large $Y$ values are anti-persistent $(s<1 / 2)$. Again, this 
Table 1. Scaling exponents $s(0)$ versus $Y$ range.

\begin{tabular}{cc}
\hline$Y$ range [nT] & $s(0)$ \\
\hline$[-5,+5]$ & $0.685 \pm 0.010$ \\
{$[-4,+4]$} & $0.640 \pm 0.010$ \\
{$[-3,+3]$} & $0.585 \pm 0.010$ \\
{$[-2,+2]$} & $0.490 \pm 0.010$ \\
\hline
\end{tabular}

different character could be the consequence of the bursty nature of the $\mathrm{AE}$ index. We will return on this point in the next Section. Furthermore, the asymmetry among the scaling exponents of positive and negative $\mathrm{AE}$ index increments in the interval $Y \in[-25,25] \mathrm{nT}$ is reflected also in a more persistent character of positive $Y$.

In Figure 8 we report the PDF master curve as obtained collapsing all the PDFs using Eq. (4) where $s$ is the rankordered spectrum $s(Y)$ reported in Fig. 7. When it is compared with the mono-scaling procedure of Fig. 3, the data collapsing is excellent for $Y<0$. The slight deviation from a perfect collapsing observed for positive $Y>120-140 \mathrm{nT}$ could be due to a nonlinear crossover between different regimes, as illustrated by Tam et al. (2010) using a different data set. This point is not investigated here.

\section{Conclusions}

In this work we investigate the multifractal nature of the $\mathrm{AE}$ index by applying the ROMA technique. Our results confirm the previous findings by Consolini et al. (1996) and the more recent results by Rypdal and Rypdal (2010) that the AE index has to be described by a multifractal model.

With respect to the previous analyses of AE index features, the ROMA technique demonstrates that the overall collapsing of the PDFs of AE index increments at different time delays $(\tau \in[4,64] \mathrm{min})$ requires a spectrum of scaling indices, the rank-ordered spectrum $s(Y)$, which is a function of the amplitude of the increments themselves. In other words, the ROMA technique is capable of identifying the specific fractal properties for increments of a certain magnitude, providing the appropriate scaling exponent associated with the collapsing of the PDFs with the scale at that increment magnitude.

In comparison with the previous analyses, the application of ROMA to positive and negative scaled increments $Y$, reveals a certain degree of asymmetry of the scaling spectrum $s(Y)$. This asymmetry has to be linked to the different physical properties of positive and negative AE index increments. As a matter of fact, positive $\mathrm{AE}$ index increments are related to an increase of auroral electrojet (and thus of auroral dissipation) while negative ones are related to the decay of auroral electrojet current intensity. The different nature of positive and negative $\mathrm{AE}$ index increments is also visible in the small discrepancy observed in the PDFs collapsing for

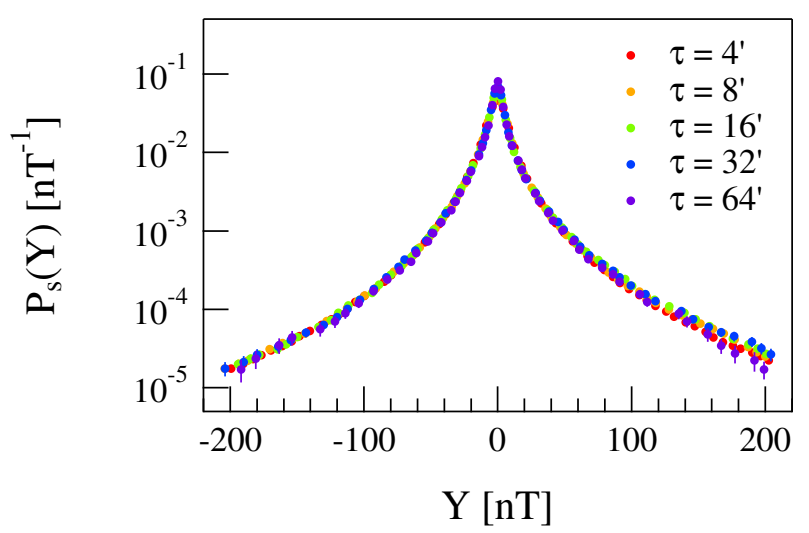

Fig. 8. Collapsing of PDFs using the discrete rank-ordered spectrum $s(Y)$ of Fig. 7 .

$Y>120-140 \mathrm{nT}$ (i.e., $\delta \mathrm{AE}(\tau)>220 \mathrm{nT}$ for $\tau=4 \mathrm{~min}$ ). A possible interpretation of this discrepancy may be a different physical origin of very large positive $\mathrm{AE}$ index increments. As well documented in several works (see e.g., Kamide and Kokubun, 1996; Consolini and De Michelis, 2005 and reference therein), the $\mathrm{AE}$ index is indeed representative of two different processes: the solar-wind directly driven convection and the impulsive unloading process. These two processes are expected to be characterized by a completely different increase of the auroral electrojet current intensity, the former being characterized by a slow and small increase of the electrojet current intensity with time, and the latter by a fast and very large increase of the $\mathrm{AE}$ index (Consolini and De Michelis, 2005). Thus, it is quite reasonable to relate the non perfect PDFs collapsing for $Y>120-140 \mathrm{nT}$ with the possible occurrence of a crossover behavior (dynamical transition to a different regime) for these very large $Y$. This point requires a more detailed analysis that will be done elsewhere in the future. In contrast, the decay mechanism seems to be characterized by a single process. This hypothesis is supported by the excellent PDF collapsing for negative $Y$ values. In conclusion, we have evidenced that the complex nature of the AE index is adequetely described in terms of a multifractal structure also by analyzing the PDF scaling. This result confirms the previous work by Consolini et al. (1996) and the recent results by Rypdal and Rypdal (2010). Furthermore, we have also provided an excellent example of the ROMA technique capability of extracting the complex nature of fluctuations (increments) of different magnitude and signs.

Acknowledgements. We are indebted with T. Chang (MIT Cambridge, USA) for the useful discussions and comments on this work. The authors thank both the National Geophysical Data Center (NGDC, Boulder, Colorado) and the World Data Center I (Kyoto, Japan) for providing data used in this work.

Edited by: H. Lamy

Reviewed by: two anonymous referees 


\section{References}

Anh, V. V., Yong J. M., and Yu, Z. G.: Stochastic modeling of the auroral electrojet index, J. Geophys. Res., 113, A10215, doi:10.1029/2007JA012851, 2008.

Benzi, R., Paladin, G., Vulpiani, A., and Parisi, G.: On the multifractal nature of fully developed turbulence and chaotic systems, J. Phys. A, Math. Gen., 17, 3521-3531 1984.

Burlaga, M. F.: Multifractal structure of the interplanetary magnetic field - Voyager 2 observations near 25 AU, 1987-1988, Geophys. Res. Lett., 18, 69-72, 1991a.

Burlaga, M. F.: Multifractal structure of speed fluctuations in recurrent streams at $1 \mathrm{AU}$ and near $6 \mathrm{AU}$, Geophys. Res. Lett., 18, 1651-1654 1991b.

Carbone, V.:, Cascade model for intermittency in fully developed magnetohydrodynamic turbulence, Phys. Rev. Lett., 71, 15461549, 1993.

Chang, T. S.: Low dimensional behaviour and symmetry breaking of stochastic systems near criticality-can these effects be observed in space and in the laboratory?, IEEE Trans. Plasma. Sci., 20, 691-694, doi:10.1109/27.199515, 1992.

Chang, T. S.: Self-organized criticality, multi-fractal spectra, sporadic localized reconnections and intermittent turbulence in magnetotail, Phys. Plasmas, 6, 4137-4145, doi:10.1063/1.873678, 1999.

Chang, T. S. and Wu, C. C.: Rank-ordered multifractal spectrum for intermittent fluctuations, Phys. Rev. E, 77, 045401, doi:10.1103/PhysRevE.77.045401, 2008.

Chang, T. S., Hankey, A., and Stanley, H. E.: Double-power scaling functions near tricritical points, Phys. Rev. B, 7, 4263-4266, doi:10.1103/PhysRevB.7.4263, 1973.

Chang, T. S., Wu, C. C., Podesta, J., Echim, M., Lamy, H., and Tam, S. W. Y.: ROMA (Rank-Ordered Multifractal Analyses) of intermittency in space plasmas - a brief tutorial review, Nonlinear Proc. Geophys., 17, 545-551, doi:10.5194/npg-17-545-2010, 2010.

Chapman S. C., Watkins, N. W., Dendy, R. O., Helander, P., and Rowlands G.: A Simple Avalanche Model as an Analogue for Magnetospheric Activity, Geophys. Res. Lett., 25, 2397-2400, doi:10.1029/98GL51700, 1998.

Chapman, S. C., Hnat, B., Rowlands, G., and Watkins, N. W.: Scaling collapse and structure functions: identifying self-affinity in finite length time series, Nonlin. Processes Geophys., 12, 767774, doi:10.5194/npg-12-767-2005, 2005.

Consolini, G.: Sandpile cellular automata and magnetospheric dynamics, in: Proc. 8th GIFCO Conference: Cosmic Physics in the Year 2000: Scientific Perspectives and New Instrumentations, edited by: Aiello, S., Iucci, N., Sironi, G., Treves, A., and Villante, U., 123, SIF Bologna, 1997.

Consolini, G.: Self-organized criticality: a new paradigm for the magnetotail dynamics, Fractals, 10, 275-283, 2002.

Consolini, G. and De Michelis, P.: Non-Gaussian distribution function of AE-index fluctuations: evidence for time intermittency, Geophys. Res. Lett., 25, 4087-4090, doi:10.1029/1998GL900073, 1998.

Consolini, G. and De Michelis, P.: A revised forest-fire cellular automaton for the nonlinear dynamics of the Earth's magnetotail, J. Atmos. Solar-Terr. Phys., 63, 1371-1377, doi:10.1016/S13646826(00)00238-8, 2001.

Consolini, G. and De Michelis, P.: Local intermittency measure analysis of $\mathrm{AE}$ index: The directly driven and unloading component, Geophys. Res. Lett., 32, L05101, doi:10.1029/2004GL022063, 2005.

Consolini, G., Marcucci, M. F., and Candidi, M.: Multifractal structure of auroral electroject index data, Phys. Rev. Lett., 76, 40824085, doi:10.1103/PhysRevLett.76.4082, 1996.

Davis, T. N. and Sugiura, M.: Auroral Electrojet Activity Index AE and its universal time variations, J. Geophys. Res., 71, 785-801, 1966.

Frisch, U.: Turbulence: The Legacy of A. N. Kolmogorov, Cambridge University Press, 1995.

Frisch, U. and Parisi, G.: Fully developed turbulence and intermittency, in: Turbulence and Predictability in geophysical Fluid Dynamics and Climate Dynamics, edited by: Ghil, M., International School of Physics "Enrico Fermi" Course 88, (North-Holland, Amsterdam), p. 84, 1985.

Halsey T. C., Jensen, M. H., Kadanoff, L. P., Procaccia, I., and Shraiman, B. I.: Fractal measures and their singularities: The characterization of strange sets, Phys. Rev. A, 33, 1141-1151, doi:10.1103/PhysRevA.33.1141, 1986.

Hergarten, S.: Self-Organized Criticality in Earth Systems, Springer Verlag, Berlin-Heidelberg, 2002.

Hnat, B., Chapman, S. C., Rowlands, G., Watkins, N. W., and Freeman, M. P.: Scaling of solar wind $\epsilon$ and AU, AL and AE indices as seen by WIND, Geophys. Res. Lett., 29, 2078, doi:10.1029/2002GL016054, 2002.

Hnat, B., Chapman, C., and Rowlands, G.: Scaling and FokkerPlanck model for fluctuations in geomagnetic indices and comparison with solar wind $\epsilon$ as seen by Wind and ACE, J. Geophys. Res., 110, doi:10.1029/2004JA010824, 2005.

Kaiser, A. and Schreiber, T.: Information transfer in continuous processes, Physica D, 166, 43-62, doi:10.1016/S01672789(02)00432-3, 2002.

Kamide, Y. and Kokubun, S.: Two-component auroral electrojet: Importance for substorm studies, J. Geophys. Res., 101, 1302713046, doi:10.1029/96JA00142, 1996.

Macek, W. M., Bruno, R., and Consolini, G.: Generalized dimensions for fluctuations in the solar wind, Phys. Rev. E, 72, 017202, doi:10.1103/PhysRevE.72.017202, 2005.

Mandelbrot, B. B.: Intermittent Turbulence in Self Similar Cascades; Divergence of High Moments and Dimensions of the Carrier, J. Fluid. Mech., 62, 331-358, doi:10.1017/S0022112074000711, 1974.

Mandelbrot, B. B.: Multifractal measures, especially for geophysicist, Pure Appl. Geophys., 131, 5-42, doi:10.1007/BF00874478, 1989.

Marsch, E., Tu, C.-Y., and Rosenbauer, H.: Multifractal scaling of the kinetic energy flux in solar wind turbulence, Ann. Geophys., 14, 259-269, doi:10.1007/s00585-996-0259-4, 1996.

Paladin, G. and Vulpiani, A.: Anomalous scaling laws in multifractal objects, Phys. Rep., 156, 147-225, doi:10.1016/03701573(87)90110-4, 1987.

Pavlos, G. P., Kyriakou, G. A., Rigas, A. G., Liatsis, P. I., Trochoutsos, P. C., and Tsonis, A. A.: Evidence for strange attractor structures in space plasma, Ann. Geophys., 10, 309-322, 1992.

Prichard, D., and Price, C. P.: Spurious dimension estimates from time series geomagnetic indices, Geophys. Res. Lett., 19, 16231626, doi:10.1029/92GL00630, 1992.

Prichard, D. and Price, C. P.: Is the AE index the result of 
nonlinear dynamics?, Geophys. Res. Lett., 20, 2817-2820, doi:10.1029/93GL03012, 1993.

Pulkkinen, A., Klimas, A., Vassiliadis, D., and Uritsky, V.: Role of stochastic fluctuations in the magnetosphere-ionosphere system: A stocastic model for the AE index variations, J. Geophys. Res., 111, A10218, doi:10.1029/2006JA011661, 2006.

Rypdal, M. and Rypdal, K.: Stochastic modelling of the AE index and its relation to fluctuations in $\mathrm{Bz}$ of the IMF on time scales shorter than substorm duration, J. Geophys. Res., 115, A11216, doi:10.1029/2010JA015463, 2010.

Roberts, D. A.: Is there a strange attractor in the magnetosphere?, J. Geophys. Res., 96, 16031-16046, doi:10.1029/91JA01088, 1991.

Shan, L. H., Goertz, C. K., and Smith, R. A.: Chaotic appearance of the AE index, Geophys. Res. Lett., 18, 147-150, doi:10.1029/90GL02477, 1991.

Sharma, A. S., Vassiliadis, D. V., and Papadopoulos, K.: Reconstruction of low-dimensional magnetospheric dynamics by singular spectrum analysis, Geophys. Res. Lett., 20, 335-338, doi:10.1029/93GL00242, 1993.

Takalo, J. and Timonen, J.: Characteristic time scale of auroral electrojet data, Geophys. Res. Lett., 21, 641-644, doi:10.1029/94GL00184, 1994.

Takalo, J., Timonen, J., and Koskinen, H.: Correlation dimension and affinity of AE data and bicolored noise, Geophys. Res. Lett., 20, 1527-1530, doi:10.1029/93GL01596, 1993.

Takalo, J., Timonen, J., and Koskinen, H.: Properties of AE data and bicolored noise, J. Geophys. Res., 99, 13239-13249, doi:10.1029/94JA00516, 1994.
Tam, S. W. Y., Chang, T., Kintner, P. M., and Klatt, E. M.: Rank-ordered multifractal analysis for intermittent fluctuations with global crossover behavior, Phys. Rev. E, 81, 036414, doi:10.1103/PhysRevE.81.036414, 2010.

Tsurutani, B. T., Goldstein, B. E., Sugiura, M., Iyemori, T., and Gonzalez, W. D.: The nonlinear response of AE to the IMF $B_{S}$ driver: a spectral break at 5 hours, Geophys. Res. Lett., 17, 279 282, doi:10.1029/GL017i003p00279, 1990.

Uritsky, V. M. and Pudovkin, M. I.: Low frequency $1 / f$-like fluctuations of the AE-index as a possible manifestation of selforganized criticality in the magnetosphere, Ann. Geophys., 16, 1580-1588, doi:10.1007/s00585-998-1580-x, 1998.

Vassiliadis, D. V., Sharma, A. S., Eastman, T. E., and Papadopoulos, K.: Low-dimensional chaos in magnetospheric activity from AE time series, Geophys. Res. Lett., 17, 1841-1844, doi:10.1029/GL017i011p01841, 1990.

Watkins, N. W., Credgington, D., Hnat, B., Chapman, S. C., Freeman, M. P., and Greenhough, J.: Towards synthesis of solar wind and geomagnetic scaling exponents: a fractional Lévy motion model, Space Sci. Rev., 121, 271-284, doi:10.1007/s11214-0064578-2, 2005.

Zimbardo, G., Greco, A., Sorriso-Valvo, L., Perri, S., Vörös, Z., Aburjania, G., Chargazia, K., and Alexandrova, O.: Magnetic turbulence in the geospace environment, Space Sci. Rev., 156, 89-134, doi:10.1007/s11214-010-9692-5, 2010. 